\title{
THE EVOLUTION OF ANISOTROPIES IN THE ELASTIC RESPONSE OF AN ELASTIC-PLASTIC MATERIAL
}

\author{
Mehrdad Negahban* and Alan S. Wineman** \\ *University of Nebraska-Lincoln and **The University of Michigan \\ (Communicated by James Casey, University of California-Berkeley)
}

\begin{abstract}
The problem of determining the change in a material's symmetries as it undergoes an elastic-plastic deformation is considered. This is interpreted as the problem of evaluating the anisotropies of the current elastic response. The discussion is presented in the context of a particular form of constitutive equation which relates the Cauchy stress to the current value of the deformation gradient and a second order tensor quantity which is a function of the deformation gradient history. A sufficient condition is established for a transformation to be a material symmetry transformation of the current elastic response. This condition relates the minimum symmetries of the current elastic response to the initial material symmetry, the given deformation history, and the structure of the constitutive equation.
\end{abstract}

\section{INTRODUCTION}

This paper is concerned with determining the changes seen in a material's symmetries after elastic-plastic deformations. Consider an initially isotropic material. There is a range of deformations from this initial state for which this material will act as an isotropic elastic solid. One can deform the material beyond this elastic limit to induce plastic flow. After inducing plastic flow, there will be a new range of deformations in which the material will only respond elastically. We call the response of the material in this range of elastic deformations its current elastic response. In general, the current elastic response of an initially isotropic material need not remain isotropic after the material undergoes an elastic-plastic deformation.

In the work presented here, we discuss the problem of determining the anisotropies of the current elastic response. In particular, we show how to evaluate the symmetries of the current elastic response function as a consequence of the material's initial symmetries, the structure of its constitutive equation, and its deformation history.

The discussion is presented within the context of a general type of constitutive equation, introduced in Section II. In this constitutive equation, the Cauchy stress depends on the current value of the deformation gradient and a second order tensor quantity, which is a functional of the deformation gradient history and which itself is considered a deformation gradient. The choice and nature of this deformation gradient will differ from model to model, but we will refer to it as the plastic deformation gradient. Thus, the Cauchy stress is assumed to be a function of the current value of the total deformation gradient and the value of the plastic deformation gradient. The constitutive equations for the stress and plastic deformation gradient are given in a general form to avoid focussing on any particular model, and to provide the most general results possible. An 
alternate structure for the constitutive equation is introduced in Section III, in which the plastic deformation gradient is replaced by the products in its polar decomposition.

The notions of a current elastic response and a yield function are defined in Section IV. In Section V, the current elastic response is expressed in terms of the constitutive equation presented in Section II. Also, the problem of finding the symmetries of the current elastic response function is formulated and discussed. It is shown that a sufficient condition for a transformation to be a material symmetry transformation of the current elastic response is that it be a member of the material's initial material symmetry group and also that it satisfy a condition given in Section V. In this manner, the minimum symmetries of the current elastic response are shown to be a consequence of the initial material symmetry, the given deformation history, and the structure of the constitutive equation for the plastic deformation gradient.

Two special cases are considered for the plastic deformation gradient in Section VI. For each case, general conclusions are drawn about the nature of the material symmetry properties of the current elastic response. These are then illustrated in Section VII for an initially isotropic material. Up to this point, the material symmetry transformations of the current elastic response have been always referred to the initial reference configuration of the material. In Sections VIII and IX, the consequence of a change to a new reference configuration is presented. In Section $\mathrm{X}$ it is shown that similar results can be obtained for a viscoelastic-plastic material.

\section{KINEMATICS, STATEMENT OF CONSTITUTIVE EQUATION}

Let $\kappa$ denote a reference configuration and let $\mathbf{X}_{K}(X)$ denote the position vector of particle $X$ in $\kappa$. Let $\mathbf{x}(t)$ denote the position vector of particle $X$ at the current time, and let the motion of a particle be described by

$$
\mathbf{x}(t)=\chi_{\kappa}\left(\mathbf{X}_{\kappa}(X), t\right)
$$

The deformation gradient evaluated at time $t$ is defined as

$$
\mathbf{F}_{\kappa}(t)=\frac{\partial \chi_{\kappa}(\mathbf{X}, t)}{\partial \mathbf{X}}
$$

Let $\mathbf{T}(t)$ denote the Cauchy stress at time $t$. A constitutive equation of the form

$$
\mathbf{T}(t)=\mathrm{T}_{\kappa}\left\{\mathbf{F}_{\kappa}(t) ; \mathbf{F}_{\kappa}^{p}(t)\right\}
$$

is assumed, where $T_{\kappa}$ is a function for the current value of the Cauchy stress tensor $\mathbf{T}(t)$ in terms of the current value of the deformation gradient $\mathbf{F}_{\kappa}(t)$ and the current value of the "plastic deformation gradient" $\mathbf{F}_{\kappa}^{p}(t)$. One will note that the common assumption made by LEE [1969] and others that the stress is a function of the "elastic deformation gradient" is a special case of this assumption since the elastic deformation gradient is normally derivable from the total deformation gradient and the plastic deformation gradient. 
It is assumed that the plastic deformation gradient can be determined by a constitutive functional ${ }^{1}$ given by

$$
\mathbf{F}_{k}^{p}(t)=\mathcal{F}_{\kappa}^{p}\left\{\begin{array}{c}
t \\
\mathbf{F}_{k}(s) \\
s=t_{s}
\end{array}\right\},
$$

where $\mathcal{F}_{\kappa}^{p}$ is a functional of the total history of the deformation gradient from a starting time $t_{s}$ to the current time $t$. Since in this development $\mathcal{F}_{\kappa}^{p}$ is a general functional, eqn (4) can be considered equivalent to the integral of the flow rule used in most theories of plasticity, but for the plastic deformation gradient.

This form of constitutive equation is selected because it generalizes many of the models of plasticity. We also observe that this model is a fully strain-based formulation.

We will interpret $\mathbf{F}_{k}^{p}(t)$ to be the deformation gradient comparing some materially significant configuration $\kappa_{p}$ to the reference configuration $\kappa$. As is commonly known, in many theories of plasticity the configuration $\kappa_{p}$ is not a true configuration. ${ }^{2}$ For each point and its neighborhood in the actual material body, $\kappa_{p}$ will contain information about the configuration of a materially significant neighborhood $\mathcal{N}_{\kappa_{p}}$ of that point. Figure 1 gives a visual representation of this interpretation.

Eqn (4) implies that for each deformation gradient history there is only one $\mathbf{F}_{k}^{p}(t)$. For example, in the case where $\kappa_{p}$ is taken to be the current stress-free configuration, by writing eqn (4) we imply that only one of the many possible stress-free configurations $^{3}$ is used to represent all such configurations. ${ }^{4}$

The functional $\mathcal{F}_{k}^{p}$ for the plastic deformation gradient is a functional in its most general sense and can be considered as the combination of a flow rule, hardening parameter, and yield function, for example. The recognition of this fact is the key to the relevance of the following development.

As a convention, in the notation we will refer to the reference configuration only the first time we introduce a new quantity and will otherwise refer to the reference configuration only when it is necessary for the development. The word configuration in this

\footnotetext{
${ }^{1}$ Some authors might disagree with the assumption that all of the plastic deformation gradient can be given by a constitutive functional (CASEY [1991], private communication).

${ }^{2}$ In many theories of plasticity the intermediate configuration $\kappa_{p}$ represents a stress-free configuration. Since a continuous configuration cannot always be found which will make the stress go to zero for all points in the material body, it is normally allowed for $\kappa_{p}$ to represent a set of disjoint configurations.

${ }^{3}$ Any rigid body rotation of a stress-free configuration is another stress-free configuration.

${ }^{4}$ As can be seen from this example, there can be many intermediate configurations $\kappa_{p}$, all representing the same physical interpretation. This results in the existence of many different $\mathbf{F}_{\kappa}^{p}(t)$ which can represent the same physical interpretation. There might be no physical justification for selecting among the many possible $\kappa_{p}$. One can consider this as a further restriction on the form of the constitutive equations. That is, if $\mathbf{F}_{\alpha}^{p}(t)$ and $\hat{\mathbf{F}}_{\kappa}^{p}(t)$ represent the deformation gradients to two physically equivalent configurations, one can require that

$$
\tau_{\kappa}\left\{\mathbf{F}_{\kappa}(t) ; \mathbf{F}_{\kappa}^{p}(t)\right\}=\mathcal{T}_{\kappa}\left\{\mathbf{F}_{\kappa}(t) ; \hat{\mathbf{F}}_{\kappa}^{p}(t)\right\}
$$

If one assumes that any rigid body rotation of $\kappa_{p}$ is another physically acceptable intermediate configuration, and one requires that the stress be form invariant to such changes of $\kappa_{p}$, this does result in a restriction on the way in which $\tau_{\kappa}$ depends on $\mathbf{F}_{\kappa}^{p}(t)$. Under such a restriction one can always write

$$
\mathbf{T}(t)=\tau_{\kappa}\left\{\mathbf{F}_{\kappa}(t) ; \mathbf{U}_{\kappa}^{p}(t)\right\},
$$

where $\mathbf{U}_{\kappa}^{p}(t)$ is a symmetric tensor given by a functional of the deformation gradient history.
} 
$\mathcal{N}_{\kappa_{p}}=$ Intermediate Configuration

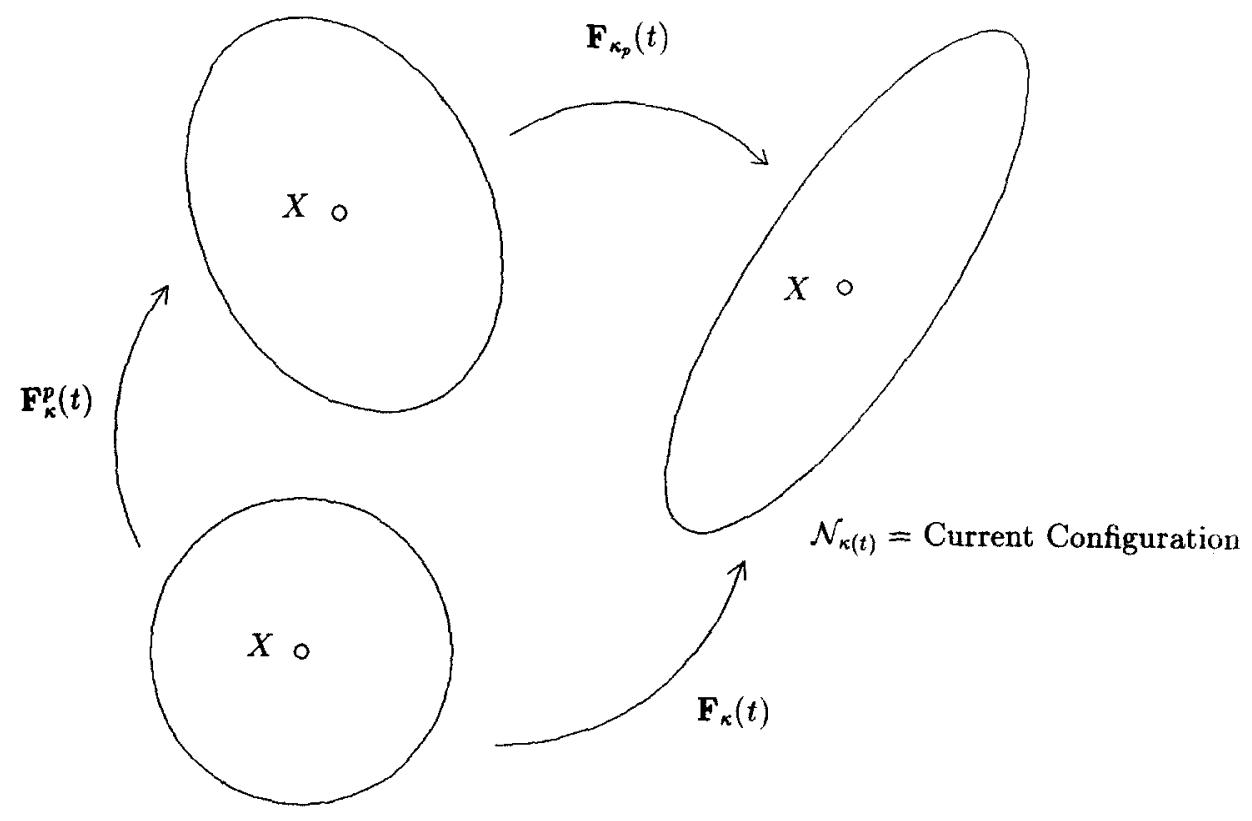

$$
\mathcal{N}_{\kappa}=\text { Reference Configuration }
$$

Fig. 1. A visual representation of $\mathbf{F}_{k}^{p}(t) . \mathbf{F}_{k}^{p}(t)$ is the deformation gradient comparing $\kappa_{p}$ to $\kappa$.

presentation will always be used to mean the configuration of the smallest neighborhood around a material point.

The constitutive eqn (3) must be subjected to the restrictions of material frame indifference. The imposition of these restrictions is discussed elsewhere (NEGAHBAN [1988]). These restrictions imply that the constitutive eqn (3) has the form

$$
\mathbf{T}(t)=\mathbf{F}(t) \mathcal{S}\left\{\mathbf{C}(t) ; \overline{\mathbf{F}}^{p}(t)\right\} \mathbf{F}^{T}(t)
$$

and that

$$
\overline{\mathbf{F}}^{p}(t)=\overline{\mathcal{T}}^{p}\left\{\begin{array}{c}
t \\
\mathbf{C}(s) \\
s=t_{s}
\end{array}\right\},
$$

where

$$
\mathbf{C}(s)=\mathbf{F}^{T}(s) \mathbf{F}(s), \quad s \in\left(t_{s}, t\right]
$$

It is assumed, for convenience, that $\overline{\mathrm{F}}^{p}(t)$ is also interpreted as a deformation gradient. The two functionals in (4) and (6) are related by 


$$
\overline{\mathcal{F}} p\left\{\begin{array}{c}
t \\
\mathbf{C}(s) \\
s=t_{s}
\end{array}\right\} \equiv \mathcal{F}^{p}\left\{\begin{array}{c}
t \\
\mathbf{U}(s) \\
s=t_{s}
\end{array}\right\}
$$

where $\mathbf{U}(s)$ is the right symmetric part of $\mathbf{F}(s)$ in its polar decomposition.

Let $g_{k}\left(t_{s}\right)$ denote the group of material symmetry transformations associated with the material at the starting time $t_{s}$ and represented with respect to the reference configuration $\kappa$ (NEGAHBan \& WiNEMAN [1989a],[1989b]) and let $\mathbf{H}$ be an element of $g\left(t_{s}\right)$. The constraint imposed by the material's initial symmetries at the starting time $t_{s}$ on the response functional of (3) is given by

$$
\tau\left\{\mathbf{F}(t) ; \mathbf{F}^{p}(t)\right\}=\tau\left\{\mathbf{F}(t) \mathbf{H} ; \mathbf{F}^{p^{*}}(t)\right\},
$$

for every $\mathbf{H} \in g\left(t_{s}\right)$, where

$$
\mathbf{F}^{p^{*}}(t)=\mathcal{F}^{p}\left\{\begin{array}{c}
t \\
\mathbf{F}(s) \mathbf{H} \\
s=t_{s}
\end{array}\right\}
$$

The restriction given in (9) must hold irrespective of the deformation gradient history $\mathbf{F}(s)$, for $s \in\left(t_{s}, t\right]$, and will henceforth be assumed without mention. The corresponding constraint on the response functional $\$$ given in (5) is

$$
\mathcal{S}\left\{\mathbf{C}(t) ; \overline{\mathbf{F}}^{p}(t)\right\}=\mathbf{H} \delta\left\{\mathbf{H}^{T} \mathbf{C}(t) \mathbf{H} ; \overline{\mathbf{F}}^{p^{*}}(t)\right\} \mathbf{H}^{T}
$$

for every $\mathbf{H} \in g\left(t_{s}\right)$, where

$$
\overline{\mathbf{F}}^{p^{*}}(t)=\overline{\mathcal{F}^{p}}\left\{\mathbf{H}^{T}{\stackrel{\mathbf{C}}{\left(s=t_{s}\right.}}_{s} \mathbf{H}\right\}
$$

\section{AN ALTERNATE STRUCTURE}

Let the polar decomposition of $\overline{\mathbf{F}}^{p}(t)$ in (5) be written as

$$
\overline{\mathbf{F}}^{p}(t)=\overline{\mathbf{R}}^{p}(t) \bar{U}^{p}(t),
$$

where $\overline{\mathbf{R}}^{p}(t)$ is orthogonal and $\overline{\mathbf{U}}^{p}(t)$ is symmetric. The uniqueness of this decomposition allows us to independently define the following functionals for $\overline{\mathbf{R}}^{p}(t)$ and $\overline{\mathbf{U}}^{p}(t)$ :

$$
\overline{\mathbf{R}}^{p}(t)=\mathbb{R}^{p}\left\{\begin{array}{c}
\mathbf{C}^{t}(s) \\
s=t_{s}
\end{array}\right\}
$$

and

$$
\overline{\mathbf{U}}^{p}(t)=\mathcal{U}^{p}\left\{\begin{array}{c}
\mathbf{C}^{\prime}(s) \\
s=t_{s}
\end{array}\right\}
$$

\footnotetext{
${ }^{5}$ See Negahban [1988] for more details.
} 
This allows us to redefine the constitutive eqn (5) such that

$$
\mathbf{T}(t)=\mathbf{F}(t) \overline{\mathcal{S}}\left\{\mathbf{C}(t) ; \overline{\mathbf{R}}^{p}(t) ; \overline{\mathbf{C}}^{p}(t)\right\} \mathbf{F}^{T}(t),
$$

where $\overline{\mathbf{C}}^{p}(t)=\overline{\mathbf{F}}^{p T}(t) \overline{\mathbf{F}}^{p}(t)=\overline{\mathbf{U}}^{p 2}(t)$, and is given by

$$
\overline{\mathbf{C}}^{p}(t)=\mathfrak{C}^{p}\left\{\begin{array}{c}
t \\
\mathbf{C}(s) \\
s=t_{s}
\end{array}\right\} \equiv \overline{\mathcal{T}}^{p T}\left\{\begin{array}{c}
t \\
\mathbf{C}(s) \\
s=t_{s}
\end{array}\right\} \overline{\mathcal{F}}^{p}\left\{\begin{array}{c}
t \\
\mathbf{C}(s) \\
s=t_{s}
\end{array}\right\} .
$$

The constraint of material symmetry on (16) is stated in a manner similar to that in (11) and can be written as

$$
\mathbf{H} \bar{\delta}\left\{\mathbf{H}^{T} \mathbf{C}(t) \mathbf{H} ; \overline{\mathbf{R}}^{p^{*}}(t) ; \overline{\mathbf{C}}^{p^{*}}(t)\right\} \mathbf{H}^{T}=\bar{\delta}\left\{\mathbf{C}(t) ; \overline{\mathbf{R}}^{p}(t) ; \overline{\mathbf{C}}^{p}(t)\right\}
$$

for every $\mathbf{H} \in g_{\kappa}\left(t_{s}\right)$, and where $\overline{\mathbf{R}}^{p}(t)$ and $\overline{\mathbf{C}}^{p}(t)$ are given by (14) and (17), and

$$
\overline{\mathbf{R}}^{p^{*}}(t)=\mathbb{R}^{p}\left\{\begin{array}{c}
t \\
\mathbf{H}^{T} \mathbf{C}(s) \mathbf{H} \\
s=t_{s}
\end{array}\right\},
$$

and

$$
\overline{\mathbf{C}}^{p^{*}}(t)=\mathfrak{e}^{p}\left\{\mathbf{H}^{T} \mathbf{C}_{s=t_{s}}^{t}(s) \mathbf{H}\right\}
$$

\section{CURRENT ELASTIC RESPONSE AND THE YIELD FUNCTION}

The idea of elastic deformations associated with the current state and that of yield surfaces are inherently interdependent. Consider a space in which every point represents a group of deformation gradients which only differ in transformations associated with rigid body motions (e.g., it can be the right Cauchy strain space). ${ }^{6}$ This will be referred to as the reduced deformation gradient space. In this space a line represents a deformation history. At each instant, a surface, called a yield surface, separates the space into points which are obtainable by "elastic deformations" and those unobtainable by such deformations. In this case "elastic" refers to deformations which will not alter the plastic deformation gradient. The point representing the current deformation gradient will always fall on or within the current yield surface. A path is drawn in this space as the neighborhood of a material particle is deformed. If this path falls on or within the yield surface, there will be no change in the yield surface. In such a case, the yield surfaces at the beginning and end points of the path will coincide. On the other hand, if this path attempts to penetrate the yield surface, the yield surface will move out to contain these deformations within its boundaries.

Motivated by the above discussion, an elastic deformation is defined as a deformation which does not alter the yield surface and the plastic deformation gradient, as opposed to an elastic-plastic deformation which will alter the yield surface and plastic deformation gradient.

\footnotetext{
${ }^{6}$ It cannot be a left Cauchy strain space.
} 
In order to provide a mathematical statement of these geometric ideas, consider a yield function which is a scalar valued functional of the deformation gradient history, $\mathbf{F}(s), s \in\left(t_{s}, t\right]$. The particular form that we use is

$$
f(t)=f\left\{\mathbf{F}(t) ; \mathbf{F}^{p}(t) ; k(t)\right\}
$$

where $\mathbf{F}^{p}(t)$ is the tensor valued functional defined in (4), and where $k(t)$ is a scalar valued functional of the deformation history given by

$$
k(t)=k\left\{\begin{array}{c}
t \\
\mathbf{F}(s) \\
s=t_{s}
\end{array}\right\},
$$

and which represents the "hardening" of the material.

We can now state the idea of elastic deformations and elastic-plastic deformations as follows:

Given an initial deformation gradient history $\mathbf{F}^{o}(s)$ for $s \in\left(t_{s}, t_{1}\right]$, then $\mathbf{F}^{p}\left(t_{1}\right)=$ $\mathcal{F}^{p}\left\{\begin{array}{c}\mathbf{F}^{t_{1}}(s) \\ s=t_{s}\end{array}\right\}$. Note that $f\left(\mathbf{F} ; \mathbf{F}^{p}\left(t_{1}\right) ; k\left(t_{1}\right)\right\}=0$ represents the yield surface at time $t_{1}$. A subsequent deformation gradient history from time $t_{1}$ to $t$ is considered to be a set of elastic deformations if

$$
f\left\{\mathbf{F}(\alpha) ; \mathbf{F}^{p}\left(t_{1}\right) ; k\left(t_{1}\right)\right\} \leq 0
$$

for every value of $\alpha \in\left(t_{1}, t\right]$. Such a history of deformations will draw a path within the yield surface associated with time $t_{1}$, and, as such, will not change the yield surface and plastic deformation gradient as time increases from $t_{1}$ to $t$. Therefore, a deformation gradient history which satisfies the conditions given in (23) will result in

$$
\mathbf{F}^{p}(\alpha)=\mathbf{F}^{p}\left(t_{1}\right) \quad \text { for all } \alpha \in\left(t_{1}, t\right]
$$

In the event that condition (23) is violated at any $\alpha \in\left(t_{1}, t\right]$, then the material will undergo an elastic-plastic deformation, and such a deformation history can result in changes in the plastic deformation gradient, hardening parameter, and, as a result, in the yield surface.

The yield function in (21) must be subjected to the constraints of frame indifference and material symmetry. However, our discussion of the evolution of anisotropies in the remainder of this paper can be carried out without determining the form imposed on (21) and (22). For the purposes of brevity in presentation, this material is presented in the appendix.

\section{CURRENT ELASTIC RESPONSE}

Consider a deformation gradient history $\mathbf{F}(s), s \in\left(t_{s}, t\right]$, whose points in the reduced deformation gradient space lie inside a yield surface for some time interval $t \in\left(t_{1}, t_{2}\right]$. In this case, condition (23) is satisfied and the values of $\mathrm{F}^{p}(t)$ and $k(t)$ remain constant over this interval. Let $\mathbf{F}^{p}$ denote the value of the plastic deformation gradient for this time interval. The discussion concerning (23) implies that $\overline{\mathbf{F}}^{p}(t), \overline{\mathbf{R}}^{p}(t)$, and $\overline{\mathbf{C}}^{p}(t)$ 
are also constant for $t \in\left(t_{1}, t_{2}\right]$. Their constant values will be denoted by $\overline{\mathbf{F}}^{p}, \overline{\mathbf{R}}^{p}$, and $\overline{\mathbf{C}}^{p}$, respectively.

Consider the restriction of the response function $\tau$ in (3) to the set of $\mathbf{F}$ which lie inside the current yield surface. This form of the constitutive equation is written as

$$
\mathbf{T}(t)=\aleph_{\kappa}\left\{\mathbf{F}_{\kappa}(t)\right\}
$$

where $\mathcal{X}$ is the current elastic response function, and which can be defined in terms of $\tau$ of (3) as

$$
\aleph\{\mathbf{F}(t)\} \equiv \tau\left\{\mathbf{F}(t) ; \mathbf{F}^{p}\right\}
$$

for the particular constant value of $\mathbf{F}^{p}$. If the constraint of frame indifference is introduced, we can write

$$
\mathbf{T}(t)=\mathbf{F}(t) \bar{\kappa}\{\mathbf{C}(t)\} \mathbf{F}^{T}(t)
$$

where $\bar{x}$ is an alternate current response function which can be written in terms of $\delta$ defined in (5) as

$$
\overline{\mathbf{x}}\{\mathbf{C}(t)\} \equiv \mathrm{S}\left\{\mathbf{C}(t) ; \overline{\mathbf{F}}^{p}\right\}
$$

for the particular value of $\overline{\mathbf{F}}^{p}$. This can also be written in terms of $\overline{\mathfrak{S}}$ given in (16) as

$$
\overline{\mathbf{X}}\{\mathbf{C}(t)\} \equiv \overline{\mathrm{s}}\left\{\mathbf{C}(t) ; \overline{\mathbf{R}}^{p} ; \overline{\mathbf{C}}^{p}\right\}
$$

for the particular constant values of $\overline{\mathbf{R}}^{p}$ and $\overline{\mathbf{C}}^{p}$.

This description of the constitutive equation is equivalent to that of nonlinear elasticity since-for the particular $\overline{\mathbf{F}}^{p}, \overline{\mathbf{R}}^{p}$, and $\overline{\mathbf{C}}^{p}$ in this interval-the response of the material only depends on the current value of the deformation gradient.

\section{V.1. Evaluation of the symmetry properties of the current elastic response function}

The problem of evaluating the symmetries of the current elastic response function of a material after it has undergone a plastic deformation can be stated as follows:

Problem Statement: Given $g\left(t_{s}\right)$, find every unimodular linear transformation $\mathbf{H}$ such that

$$
\mathbf{T}(t)=\kappa\{\mathbf{F}(t)\}=\aleph\{\mathbf{F}(t) \mathbf{H}\}
$$

irrespective of the particular value of $\mathbf{F}(t)$. That is, given the initial material symmetry, find the invariance properties of $\$$ for the current elastic region.

The problem can be restated in terms of the current elastic response function $\bar{x}$ as follows. 
Alternate Statement: Given $g\left(t_{s}\right)$, find every unimodular linear transformation $\mathbf{H}$ which satisfies the condition

$$
\mathbf{H} \overline{\mathbf{x}}\left\{\mathbf{H}^{T} \mathbf{C}(t) \mathbf{H}\right\} \mathbf{H}^{T}=\overline{\mathbf{x}}\{\mathbf{C}(t)\},
$$

irrespective of the value of $\mathbf{C}(t)$.

It is convenient to restate (31) using the definition of $\bar{x}$ in terms of $\bar{s}$ in (29): Given $g\left(t_{s}\right)$, find every unimodular linear transformation $\mathbf{H}$ which satisfies the condition

$$
\mathbf{H} \overline{\mathcal{S}}\left\{\mathbf{H}^{T} \mathbf{C}(t) \mathbf{H} ; \overline{\mathbf{R}}^{p} ; \overline{\mathbf{C}}^{p}\right\} \mathbf{H}^{T}=\overline{\mathcal{S}}\left\{\mathbf{C}(t) ; \overline{\mathbf{R}}^{p} ; \overline{\mathbf{C}}^{p}\right\} .
$$

In order to arrive at a solution, recall that the material symmetry restriction on $\bar{\AA}$ due to the initial symmetries of the material at the starting time $t_{s}$ is stated in (18). It is repeated here for convenience:

$$
\mathbf{H} \overline{\mathcal{S}}\left\{\mathbf{H}^{T} \mathbf{C}(t) \mathbf{H} ; \overline{\mathbf{R}}^{p^{*}}(t) ; \overline{\mathbf{C}}^{p^{*}}(t)\right\} \mathbf{H}^{T}=\overline{\mathcal{S}}\left\{\mathbf{C}(t) ; \overline{\mathbf{R}}^{p}(t) ; \overline{\mathbf{C}}^{p}(t)\right\},
$$

for every $\mathbf{H} \in g\left(t_{s}\right)$, and where $\overline{\mathbf{R}}^{p^{*}}(t)$ and $\overline{\mathbf{C}}^{p^{*}}(t)$ are given in (19) and (20).

A comparison of (32) and (33) leads to the following solution:

Solution: A sufficient condition for a unimodular transformation $\mathbf{H}$ to represent a symmetry of the current elastic response function is that $\mathbf{H} \in g\left(t_{s}\right)$ and also satisfies the conditions

$$
\mathcal{R}^{p}\left\{\begin{array}{c}
\mathbf{C}(s) \\
s=t_{s}
\end{array}\right\}=\mathbb{R}^{p}\left\{\underset{\substack{\mathbf{H}^{T} \\
\mathbf{C}(s) t_{s}}}{t} \mathbf{H}\right\}
$$

and

$$
\mathfrak{e}^{p}\left\{\underset{s=t_{s}}{\mathbf{C}^{t}(s)}\right\}=\mathfrak{e}^{p}\left\{\mathbf{H}^{T} \underset{s=t_{s}}{\mathbf{C}^{t}(s) \mathbf{H}}\right\} .
$$

Therefore, the problem of following the evolution of anisotropies in the elastic response of the material has become the new problem of solving for those $\mathbf{H} \in g\left(t_{s}\right)$ which satisfy (34) and (35).

Comment 1: The solution presented here is for the most general function $\overline{\mathrm{s}}$ and functionals $\mathbb{R}^{p}$ and $\mathfrak{C}^{p}$. If $\overline{\mathrm{S}}, \boldsymbol{R}^{p}$, and $\mathfrak{C}^{p}$ are given more explicit or simpler structures, there might be other transformations $H$ which also satisfy (32). That is, the results obtained here are common to all theories which are of the form given in (16). Therefore, the solution presented here will identify the smallest group of symmetry transformations of the function $\bar{x}$ which are common to all simple materials whose constitutive equation can be written in the form given by (16).

Comment 2: The transformations $H$ for which (34) and (35) are satisfied depend on the deformation history $\mathrm{C}(s), s \in\left(t_{s}, t\right]$, and also on the explicit forms of the functionals $\mathbb{R}^{p}$ and $\mathfrak{C}^{p}$.

The group of transformations representing the symmetries of the current elastic response function of the material at time $t$ will be denoted by $g_{x}^{e}(t)$. In this notation and 
in the following presentation, the parameter $t$ will refer to the current yield surface and the elastic response within the current yield surface as opposed to the actual current time.

The solution can be restated as follows.

Solution: $g^{e}(t)$ will always satisfy

$$
g^{e}(t) \supseteq g^{p}(t) \cap g\left(t_{s}\right),
$$

where $g^{p}(t)$ is a mathematical group defined as

$$
g^{p}(t)=\left\{\mathbf{H} \mid \overline{\mathbf{R}}^{p^{*}}(t)=\overline{\mathbf{R}}^{p}(t), \overline{\mathbf{C}}^{p^{*}}(t)=\overline{\mathbf{C}}^{p}(t), \text { and } \operatorname{det}(\mathbf{H})= \pm 1\right\},
$$

for the particular history under consideration.

An explicit statement of the values of $\mathbf{H}$ which satisfy (31) can be given once more structure is given to the functionals $\mathbb{R}^{p}$ and $\mathrm{C}^{p}$. Since $\mathbb{R}^{p}$ is an orthogonal tensor valued functional of its argument, there is an orthogonal tensor valued functional $\nu$ such that

$$
\mathbb{R}^{p}\left\{\underset{\mathbf{H}^{T} \mathbf{C}(s) \mathbf{H}}{s=t_{s}}\right\}=\nu\left\{\underset{\mathbf{H} ; \underset{\mathbf{C}(s)}{t=t_{s}}}{\mathbf{C}_{s=t_{s}}}\right\} \mathbb{R}^{p}\left\{\begin{array}{c}
\mathbf{C}^{\prime}(s) \\
s=t_{s}
\end{array}\right\}
$$

for every $\mathbf{H} \in g\left(t_{s}\right)$. Furthermore, since $\mathcal{C}^{p}$ is a symmetric tensor valued functional, there is a tensor valued functional $\mu$ such that

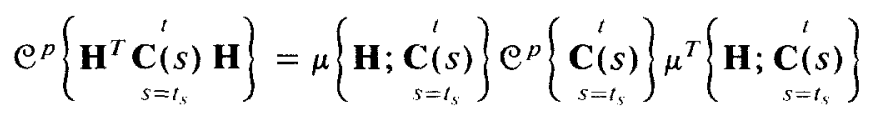

for every $\mathbf{H} \in g\left(t_{s}\right)$. $^{7}$

As examples, we consider the following two special cases:

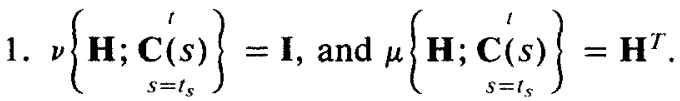

2. $\nu\left\{\begin{array}{c}\mathbf{H} ; \mathbf{C}(s) \\ s=t_{s}\end{array}\right\}=\mathbf{I}$, and $\mu\left\{\begin{array}{c}\mathbf{H} ; \underset{\mathbf{C}(s)}{s=t_{s}} \\ \mathbf{c}\end{array}\right\}=\mathbf{I}$.

In each case, we show how the assumptions influence the structure of the functionals, and how they influence the elastic response of the material. The results will be presented for the case of an initially isotropic material, even though this procedure is not limited to such materials.

\footnotetext{
${ }^{7}$ Any two symmetric positive definite matrices $\mathbf{A}$ and $\mathbf{B}$ can be written as $\mathbf{A}=\mathbf{D B D} \mathbf{D}^{\mathbf{T}}$ for some matrix $\mathbf{D}$. The symmetry of $\mathbf{A}$ and $\mathbf{B}$ guarantees the existence of $\mathbf{A}_{d}=\mathbf{P A P} \mathbf{P}^{T}$ and $\mathbf{B}_{d}=\mathbf{Q R Q} \mathbf{Q}^{T}$, where $\mathbf{A}_{d}$ is the diagonal matrix of the eigenvalues of $\mathbf{A}, \mathbf{B}_{d}$ is the diagonal matrix of the eigenvalues of $\mathbf{B}$, and $\mathbf{P}$ and $\mathbf{Q}$ are orthogonal. Let $\mathbf{A}_{d}=\operatorname{DIAG}\left(a_{1}, a_{2}, a_{3}\right)$ and $\mathbf{B}_{d}=\operatorname{DIAG}\left(b_{1}, b_{2}, b_{3}\right)$. Since $\mathbf{A}$ is not singular, one can always write $\mathbf{B}_{d}=\mathbf{J}^{T} \mathbf{A}_{d} \mathbf{J}$, where $\mathbf{J}$ is a diagonal matrix given by $\mathbf{J}=D I A G\left(\sqrt{b_{1} / a_{1}}, \sqrt{b_{2} / a_{2}}, \sqrt{b_{3} / a_{3}}\right)$. This results in the relation $\mathbf{B}=\mathbf{Q}^{T} \mathbf{J}^{T} \mathbf{P A P} \mathbf{P}^{T} \mathbf{J Q}$ which gives $\mathbf{D}=\mathbf{Q}^{T} \mathbf{J}^{T} \mathbf{P}$.
} 


\section{SPECIAL CASES}

Before proceeding further, it will be useful to summarize the assumptions as follows:

1. The constitutive equation for the Cauchy stress is given by

$$
\mathbf{T}(t)=\mathbf{F}(t) \overline{\mathcal{S}}\left\{\mathbf{C}(t) ; \overline{\mathbf{R}}^{p}(t) ; \overline{\mathbf{C}}^{p}(t)\right\} \mathbf{F}^{T}(t),
$$

where $\overline{\mathbf{R}}^{p}(t)=\mathbb{R}^{p}\left\{\begin{array}{c}t \\ \mathbf{C}(s) \\ s=t_{s}\end{array}\right\}$ is orthogonal, and $\overline{\mathbf{C}}^{p}(t)=\mathbb{C}^{p}\left\{\begin{array}{c}t \\ \mathbf{C}(s) \\ s=t_{s}\end{array}\right\}$ is symmetric.

2. The yield function is given as

$$
f(t)=\bar{f}\left\{\mathbf{C}(t) ; \overline{\mathbf{R}}^{p}(t) ; \overline{\mathbf{C}}^{p}(t) ; \bar{k}(t)\right\},
$$

and $\bar{k}(t)=\bar{k}_{\kappa}\left\{\begin{array}{c}t \\ \mathbf{C}(s) \\ s=t_{s}\end{array}\right\}$ (see the appendix for details).

3. For a fixed yield surface, $\overline{\mathbf{R}}^{p}(t)$ and $\overline{\mathbf{C}}^{p}(t)$ are both constants, denoted respectively as $\overline{\mathbf{R}}^{p}$ and $\overline{\mathbf{C}}^{p}$. For deformations within this yield surface, the elastic response is given as

$$
\mathbf{T}(t)=\mathbf{F}(t) \bar{\kappa}\{\mathbf{C}(t)\} \mathbf{F}^{T}(t)
$$

where

$$
\overline{\boldsymbol{X}}\{\mathbf{C}(t)\} \equiv \overline{\mathcal{S}}\left\{\mathbf{C}(t) ; \overline{\mathbf{R}}^{p} ; \overline{\mathbf{C}}^{p}\right\}
$$

It will henceforth be assumed that for all times and histories

$$
\overline{\mathbf{R}}^{p}(t)=\mathcal{R}^{p}\left\{\begin{array}{c}
t \\
\mathbf{C}(s) \\
s=t_{s}
\end{array}\right\}=\mathbf{I}^{8}
$$

Then (40) and (43) become

$$
\mathbf{T}(t)=\mathbf{F}(t) \bar{\S}\left\{\mathbf{C}(t) ; \mathbf{I} ; \overline{\mathbf{C}}^{p}(t)\right\} \mathbf{F}^{T}(t),
$$

and

$$
\overline{\boldsymbol{X}}\{\mathbf{C}(t)\}=\overline{\mathcal{S}}\left\{\mathbf{C}(t) ; \mathbf{I} ; \overline{\mathbf{C}}^{p}\right\}
$$

It is worth mentioning at this point that a relation can be established between $\overline{\mathbf{R}}_{\kappa}^{p}(t)$ and $\overline{\mathbf{R}}_{\kappa^{\prime}}^{p}(t)$, where $\kappa^{\prime}$ denotes a new reference configuration. Even though we have

\footnotetext{
${ }^{8}$ This assumption is not in contradiction with any invariance conditions imposed by material frame indifference on the stress. These conditions are all satisfied directly in Section II. As has been shown by CASEY and NAGHDI [1980], [1981], it is possible to violate invariance conditions associated with frame indifference if restrictions are put on both $\mathbf{F}^{e}$ and $\mathbf{F}^{p}$ and if it is also assumed that $\mathbf{F}=\mathbf{F}^{e} \mathbf{F}^{p}$. This is not the case in our presentation. (Also see footnote 4 of Section II.)
} 
made the assumption in (44) that $\overline{\mathbf{R}}_{\kappa}^{p}(t)$ is always equal to the identity, it can be shown that $\overline{\mathbf{R}}_{\kappa^{\prime}}^{p}(t) \neq \mathbf{I}$, in general (NEGAHBAN [1988]).

VI.1. Case $1: \nu=\mathbf{I}, \mu=\mathbf{H}^{T}$

With this assumption applied to (38) and (39), the statement of the restrictions imposed by the material's initial symmetry, given in (18), becomes

$$
\mathbf{H} \overline{\mathcal{S}}\left\{\mathbf{H}^{T} \mathbf{C}(t) \mathbf{H} ; \mathbf{I} ; \mathbf{H}^{T} \overline{\mathbf{C}}^{p}(t) \mathbf{H}\right\} \mathbf{H}^{T}=\overline{\mathcal{S}}\left\{\mathbf{C}(t) ; \mathbf{I} ; \overline{\mathbf{C}}^{p}(t)\right\}
$$

for every $\mathbf{H} \in g\left(t_{s}\right)$. The problem of finding the symmetries of the current elastic response function $\overline{\boldsymbol{x}}$, as defined by (32), can be restated as: Find every unimodular $\mathbf{H}$ which results in

$$
\mathbf{H} \overline{\mathcal{S}}\left\{\mathbf{H}^{T} \mathbf{C}(t) \mathbf{H} ; \mathbf{I} ; \overline{\mathbf{C}}^{p}\right\} \mathbf{H}^{T}=\bar{\S}\left\{\mathbf{C}(t) ; \mathbf{I} ; \overline{\mathbf{C}}^{p}\right\}
$$

for all values of $\mathbf{C}(t)$.

A comparison of (47) and (48) shows that $\mathbf{H}$ will be a symmetry of $\bar{X}$ if $\mathbf{H} \in g\left(t_{s}\right)$ and satisfies the equation

$$
\mathbf{H}^{T} \overline{\mathbf{C}}^{p} \mathbf{H}=\overline{\mathbf{C}}^{p}
$$

for the particular value of $\overline{\mathbf{C}}^{p}$ corresponding to $\overline{\mathrm{x}}$. The transformations which satisfy (49) form a group, and are denoted by $g^{p}(t)$.

The solution to the problem of evaluating the symmetries of the elastic response for the case currently under consideration can now be stated as:

$$
g^{e}(t) \supseteq g\left(t_{s}\right) \cap g^{p}(t)
$$

where

$$
g\left(t_{s}\right) \cap g^{p}(t)=\left\{\mathbf{H} \mid \mathbf{H} \in g\left(t_{s}\right) \text { and } \mathbf{H}^{T} \overline{\mathbf{C}}^{p} \mathbf{H}=\overline{\mathbf{C}}^{p}\right\}
$$

Hence, when the value of $\overline{\mathbf{C}}^{p}$ corresponding to the current yield surface is known, the problem is reduced to solving for all $\mathbf{H}$ which satisfy (51).

\section{VI.2. Case 2: $\nu=\mathbf{I}, \mu=\mathbf{I}$}

With this assumption applied to (38) and (39), the statement of the restriction imposed by the material's initial symmetry, given in (18), becomes

$$
\mathbf{H} \overline{\mathbf{s}}\left\{\mathbf{H}^{T} \mathbf{C}(t) \mathbf{H} ; \mathbf{I} ; \overline{\mathbf{C}}^{p}\right\} \mathbf{H}^{T}=\overline{\mathfrak{s}}\left\{\mathbf{C}(t) ; \mathbf{I} ; \overline{\mathbf{C}}^{p}\right\}
$$

for every $\mathbf{H} \in g\left(t_{s}\right)$. A comparison of (48) and (52) leads to the conclusion that each symmetry transformation $H \in g\left(t_{s}\right)$ is also a symmetry transformation of the current elastic response function $\bar{x}$. In other terms, for the present case

$$
g^{e}(t) \supseteq g\left(t_{s}\right)
$$


It must be noted that both $g^{e}(t)$ and $g\left(t_{s}\right)$ are groups representing the symmetry in terms of linear transformations of the same initial reference configuration $\kappa$.

\section{INITIALLY ISOTROPIC MATERIALS}

For materials which are initially isotropic, $g\left(t_{s}\right)=\mathcal{O} \equiv$ [the full group of orthogonal transformations\}.

VII.1. Case $1: \nu=\mathbf{I}$ and $\mu=\mathbf{H}^{T}$

The condition (39) becomes

$$
\mathbf{H}^{T} \mathfrak{C}^{p}\left\{\begin{array}{c}
\mathbf{C}^{t}(s) \\
s=t_{s}
\end{array}\right\} \mathbf{H}=\mathfrak{e}^{p}\left\{\underset{\mathbf{H}^{T} \mathbf{C}(s) \mathbf{H}}{s=t_{s}}\right\}
$$

for every $\mathbf{H} \in g\left(t_{s}\right)=\mathcal{O}$ and every arbitrary $\mathbf{C}(s)$ and $t$. This constrains $\mathcal{C}^{p}$ to the form of an isotropic functional of the history of $\mathbf{C}(s)$ (in discussing isotropic materials, we use the terminology "isotropic function" and "isotropic invariants." This is consistent with common usage in the literature and as given in SPENCER [1975]). Using the representation developed by WINEMaN and PIPKIN [1964] for such functionals, the constitutive equation for $\overline{\mathbf{C}}^{p}(t)$ can be written as

$$
\overline{\mathbf{C}}^{p}(t)=\mathfrak{e}^{p}\left\{\begin{array}{c}
t \\
\mathbf{C}(s) \\
s=t_{s}
\end{array}\right\}=\sum_{i=0}^{5} L^{(i)}\left\{\mathbf{J}^{(i)} ; I_{1}, \ldots, I_{6}\right\},
$$

where each $L^{(i)}$ is a functional which is linear in the argument $\mathbf{J}^{(i)}$, where

$$
\begin{gathered}
\mathbf{J}^{(0)}=\mathbf{I} \\
\mathbf{J}^{(i)}=\frac{1}{2}\left(\Pi^{(i)}+\Pi^{(i)^{T}}\right), \quad i=1, \ldots, 5 \\
\Pi^{(i)}=\mathbf{C}\left(\psi_{1}\right) \ldots \mathbf{C}\left(\psi_{i}\right), \quad \text { for } t_{s}<\psi_{\alpha} \leq t,
\end{gathered}
$$

and where $I_{1}, \ldots, I_{6}$ are scalar invariants of $\mathbf{C}(s)$ defined by

$$
\begin{gathered}
I_{i}=\operatorname{tr}\left[\mathbf{C}\left(\hat{\psi}_{1}\right) \mathbf{C}\left(\hat{\psi}_{2}\right) \ldots \mathbf{C}\left(\hat{\psi}_{i}\right)\right], \quad i=1,2, \ldots, 6 \\
t_{s}<\hat{\psi}_{i} \leq t,
\end{gathered}
$$

and where tr stands for the trace operation. Each functional $L^{(i)}$ depends on all values of its arguments as the time variables $\psi_{i}$ and $\hat{\psi}_{i}$ vary in the indicated intervals. Even though this representation is more general than that normally used for theories of plasticity, any representation of plasticity which satisfies (54) should have a $\mathfrak{C}^{p}$ expressible in this form.

In the constitutive eqn (45), the response functional $\overline{\mathcal{S}}$ must satisfy the material symmetry restriction in (47) for every $\mathbf{H} \in g\left(t_{s}\right)=\mathcal{O}$. Therefore, $\bar{\delta}$ is an isotropic function of its two symmetric tensor arguments $\mathbf{C}(t)$ and $\overline{\mathbf{C}}^{p}(t)$. Its representation, given by the results of RIVIIN and ERICKSON [1955] on isotropic functions of two symmetric second order tensors, is 


$$
\begin{aligned}
\bar{s}\left\{\mathbf{C}(t) ; \mathbf{I} ; \overline{\mathbf{C}}^{p}(t)\right\}= & a_{1} \mathbf{I}+a_{2} \mathbf{C}(t)+a_{3} \mathbf{C}^{2}(t)+a_{4} \overline{\mathbf{C}}^{p}(t) \\
& +a_{5} \overline{\mathbf{C}}^{p 2}(t)+a_{6}\left[\mathbf{C}(t) \overline{\mathbf{C}}^{p}(t)+\overline{\mathbf{C}}^{p}(t) \mathbf{C}(t)\right] \\
& +a_{7}\left[\mathbf{C}^{2}(t) \overline{\mathbf{C}}^{p}(t)+\overline{\mathbf{C}}^{p}(t) \mathbf{C}^{2}(t)\right]+a_{8}\left[\overline{\mathbf{C}}^{p 2}(t) \mathbf{C}(t)+\mathbf{C}(t) \overline{\mathbf{C}}^{p 2}(t)\right] \\
& +a_{9}\left[\mathbf{C}^{2}(t) \overline{\mathbf{C}}^{p 2}(t)+\overline{\mathbf{C}}^{p 2}(t) \mathbf{C}^{2}(t)\right]
\end{aligned}
$$

where $a_{1}, \ldots, a_{9}$ are functions of the 10 isotropic invariants of $\mathbf{C}(t)$ and $\overline{\mathbf{C}}^{p}(t)$ given by

$$
\begin{gathered}
I_{1}^{*}=\operatorname{tr}[\mathbf{C}(t)], \quad I_{2}^{*}=\operatorname{tr}\left[\mathbf{C}^{2}(t)\right], \quad I_{3}^{*}=\operatorname{tr}\left[\mathbf{C}^{3}(t)\right], \\
I_{4}^{*}=\operatorname{tr}\left[\overline{\mathbf{C}}^{p}(t)\right], \quad I_{5}^{*}=\operatorname{tr}\left[\overline{\mathbf{C}}^{p 2}(t)\right], \quad I_{6}^{*}=\operatorname{tr}\left[\overline{\mathbf{C}}^{p 3}(t)\right], \\
I_{7}^{*}=\operatorname{tr}\left[\mathbf{C}(t) \overline{\mathbf{C}}^{p}(t)\right], \quad I_{8}^{*}=\operatorname{tr}\left[\mathbf{C}(t) \overline{\mathbf{C}}^{p 2}(t)\right], \quad I_{9}^{*}=\operatorname{tr}\left[\mathbf{C}^{2}(t) \overline{\mathbf{C}}^{p}(t)\right], \\
I_{10}^{*}=\operatorname{tr}\left[\mathbf{C}^{2}(t) \overline{\mathbf{C}}^{p 2}(t)\right] .
\end{gathered}
$$

According to (42) and (43), the current elastic response function $\bar{x}$ is given by $\overline{\mathcal{S}}$ in (58), in which $\overline{\mathbf{C}}^{p}(t)$ has a constant value, denoted by $\overline{\mathbf{C}}^{p}$. Thus,

$$
\mathbf{T}(t)=\mathbf{F}(t) \bar{\aleph}\{\mathbf{C}(t)\} \mathbf{F}^{T}(t)
$$

in which

$$
\begin{aligned}
\overline{\boldsymbol{X}}\{\mathbf{C}(t)\}= & a_{1} \mathbf{I}+a_{2} \mathbf{C}(t)+a_{3} \mathbf{C}^{2}(t)+a_{4} \overline{\mathbf{C}}^{p}+a_{5} \overline{\mathbf{C}}^{p 2} \\
& +a_{6}\left[\mathbf{C}(t) \overline{\mathbf{C}}^{p}+\overline{\mathbf{C}}^{p} \mathbf{C}(t)\right]+a_{7}\left[\mathbf{C}^{2}(t) \overline{\mathbf{C}}^{p}+\overline{\mathbf{C}}^{p} \mathbf{C}^{2}(t)\right] \\
& +a_{8}\left[\overline{\mathbf{C}}^{p 2} \mathbf{C}(t)+\mathbf{C}(t) \overline{\mathbf{C}}^{p 2}\right]+a_{9}\left[\mathbf{C}^{2}(t) \overline{\mathbf{C}}^{p 2}+\overline{\mathbf{C}}^{p 2} \mathbf{C}^{2}(t)\right]
\end{aligned}
$$

Note that, by $(51), \bar{x}$ has the property

$$
\mathbf{H} \overline{\boldsymbol{x}}\left\{\mathbf{H}^{T} \mathbf{C}(t) \mathbf{H}\right\} \mathbf{H}^{T}=\overline{\boldsymbol{\kappa}}\{\mathbf{C}(t)\}
$$

for every $\mathbf{H} \in g^{p}(t) \cap g\left(t_{s}\right)$.

The structure of the group of transformations $g^{p}(t) \cap g_{\kappa}\left(t_{s}\right)$ has been discussed in NEGAHBAN and WinEMAN [1989a]. It is shown there that the material symmetry of the current elastic response function depends on the principal values of $\overline{\mathbf{C}}^{p}$, denoted as $\lambda_{p 1}, \lambda_{p 2}, \lambda_{p 3}$, in the following manner:

The transformations in $g^{p}(t) \cap g\left(t_{s}\right)$ are those associated with

1. Isotropy if $\lambda_{p 1}=\lambda_{p 2}=\lambda_{p 3}$,

2. Transverse isotropy if $\lambda_{p 1}=\lambda_{p 2} \neq \lambda_{p 3}$,

3. Orthotropy if $\lambda_{p 1} \neq \lambda_{p 2} \neq \lambda_{p 3} \neq \lambda_{p 1}$.

The preferred axis of transverse isotropy is along the principal axis of $\overline{\mathbf{C}}^{p}$ associated with the principal value $\lambda_{p 3}$. The preferred axes of orthotropy are along the principal axes of $\overline{\mathbf{C}}^{p}$.

In short, the symmetry of the current elastic response function of the material will at least be that of isotropy, transverse isotropy, or orthotropy, depending on the eigenvalues of $\overline{\mathbf{C}}^{p}$ (no other choices exist for this material). 
VII.2. Case 2: $\nu=\mathrm{I}$ and $\mu=\mathrm{I}$

In this case, condition (39) becomes

$$
\mathfrak{e}^{p}\left\{\begin{array}{c}
\mathbf{C}^{\prime}(s) \\
s=t_{s}
\end{array}\right\}=\mathfrak{e}^{p}\left\{\begin{array}{c}
\mathbf{H}^{T} \\
\mathbf{C}(s) \mathbf{H} \\
s=t_{s}
\end{array}\right\}
$$

for every $\mathbf{H} \in g\left(t_{s}\right)=\mathcal{O}$. Therefore, $\mathfrak{e}^{p}$ must be a functional of the history of the six isotropic invariants of $\mathrm{C}(s)$. That is,

$$
\overline{\mathbf{C}}^{p}(t)=\mathfrak{e}^{p}\left\{I_{1}, \ldots, I_{6}\right\}
$$

where $I_{1}, \ldots, I_{6}$ are given in (57).

In constitutive eqn (45), the response functional $\bar{\delta}$ must satisfy the material symmetry restriction (52) for every $\mathbf{H} \in g\left(t_{s}\right)=\mathcal{O}$. Thus, $\bar{\S}$ is an isotropic function of only $\mathbf{C}(t)$. It is well known, in this case, that the most general constitutive equation has the form

$$
\mathbf{T}(t)=p_{0} \mathbf{I}+p_{1} \mathbf{B}(t)+p_{2} \mathbf{B}^{2}(t)
$$

where $\mathbf{B}(t)=\mathbf{F}(t) \mathbf{F}^{T}(t)$. The three scalars $p_{1}, p_{2}$, and $p_{3}$ will be functions of the three isotropic invariants of $\mathbf{B}(t)$ and the six independent components of $\overline{\mathbf{C}}^{p}(t)$. It is seen that the principal directions of the Cauchy stress will be the same as that of the left Cauchy strain $\mathbf{B}(t)$.

Recall that the current elastic response function is obtained from the general response function when $\overline{\mathbf{C}}^{p}(t)$ has a constant value. In this case, the current elastic constitutive equation will be given by an equation similar to (65) in which $p_{1}, p_{2}, p_{3}$ are functions of only the three isotropic invariants of $\mathbf{B}(t)$. This representation for the current elastic response function illustrates the statement in (53) that $g_{\kappa}^{e}(t) \supseteq g\left(t_{s}\right)=\mathcal{O}$.

In concluding this section we make the following comments and comparisons about cases 1 and 2 .

1. The principal directions of the Cauchy stress, $\mathbf{T}(t)$, and the left Cauchy strain, ${ }^{9}$ $\mathbf{B}(t)=\mathbf{F}(t) \mathbf{F}^{T}(t)$, do not coincide for the first case (in general), but always coincide for the second case.

2. Consider a history of deformations in which the material returns to the reference configuration $\kappa$, corresponding to $\mathbf{B}(t)=\mathbf{I}$. The state of stress corresponding to this configuration could have any structure for the first case under consideration, but it can only correspond to hydrostatic pressure or tension for the second case.

3. Because of the similarity of constitutive eqn (65) and that of nonlinear isotropic elasticity, universal relations which have been obtained for nonlinear elasticity apply directly for the second case, and can be used for experimental verification of this model.

\footnotetext{
${ }^{9}$ The left Cauchy strain is measured relative to the initial reference configuration $\kappa$ and not the intermediate configuration $\kappa_{p}$.
} 
4. The second case allows a distinct possibility that there exists a stress-free configuration corresponding to an equal triaxial extension from the reference configuration $\kappa$ (i.e., $\mathbf{B}=\lambda \mathbf{I}$ ).

\section{CHANGE OF REFERENCE CONFIGURATION-GENERAL MATERIAL}

It is common in plasticity to write the constitutive equation for stress in terms of the "elastic strain" (strain measured with respect to the intermediate configuration $\kappa_{p}$ and not with respect to the initial reference configuration $\kappa$ ). To show how our results compare with these models, we will rewrite the above constitutive equations relative to the intermediate configuration $\kappa_{p}$. This will be accomplished by a proper change of reference configuration.

As distinction between reference configurations is necessary in this development, we will add the reference configuration to all variables of the development.

To simplify the presentation we assume $\mathbf{F}_{k}^{p}(t)$ to be the same as $\overline{\mathbf{F}}_{k}^{p}(t)$. This eliminates the possibility of $\mathbf{F}_{k}^{p}(t)$ changing with the superposition of rigid body motions on the deformation history.

The current response function $\aleph_{\kappa}$ is associated with a specific yield function and a specific value of $\overline{\mathbf{F}}_{\kappa}^{p}(t)$. The argument of $\aleph_{\kappa}$ is the deformation gradient $\mathbf{F}_{\kappa}(t)$ which compares the current configuration to the reference configuration $\kappa$. The transformations $\mathbf{H}$ which describe the symmetries of $\boldsymbol{x}_{k}$ are transformations of the reference configuration $\kappa$ to mechanically equivalent ones.

We now consider a change from the initial reference configuration $\kappa$ to the configuration $\kappa_{p}$ associated with the current yield function. There will be a change in the form of the current response function. There will also be different material symmetry transformations representing the same symmetries, but which now are transformations from the new reference configuration to mechanically equivalent ones.

In Section II $\overline{\mathbf{F}}_{\kappa}^{p}(t)$ was interpreted to be a deformation gradient which compares $\kappa_{p}$ to the initial reference configuration $\kappa$. Since a constant value of $\overline{\mathbf{F}}_{k}^{p}$ is associated with a fixed yield surface, the configuration $\kappa_{p}$ can also be taken as constant (up to a pure translation). In this section, we will rewrite the response functionals with respect to this configuration, and discuss the corresponding material symmetry transformations.

The deformation gradient of the current configuration with respect to configuration $\kappa_{p}$ is defined as

$$
\mathbf{F}_{\kappa_{p}}(t)=\frac{\partial \chi_{\kappa_{p}}\left(\mathbf{X}_{\kappa_{p}} ; t\right)}{\partial \mathbf{X}_{\kappa_{p}}}
$$

where $\mathbf{X}_{\kappa_{p}}$ is the position in configuration $\kappa_{p}$ of the particle which had position $\mathbf{X}_{\kappa}$ in configuration $\kappa .{ }^{10}$ Deformation gradients $\mathbf{F}_{\kappa_{p}}(t)$ and $\mathbf{F}_{k}(t)$, defined in $(2)$, are related by

$$
\mathbf{F}_{\kappa}(t)=\mathbf{F}_{\kappa p}(t) \overline{\mathbf{F}}_{\kappa}^{p},
$$

\footnotetext{
${ }^{10}$ As stated in Section II, since the intermediate configuration is not a real configuration in most theories of plasticity, we consider $\kappa_{p}$ to be a set of configurations each representing a neighborhood.
} 
where, according to the interpretation discussed in Section 2,

$$
\overline{\mathbf{F}}_{\kappa}^{p}=\frac{\partial \mathbf{X}_{\kappa_{p}}}{\partial \mathbf{X}_{\kappa}}
$$

The constitutive equation in (25) establishes the relation between the current response function $\aleph_{k}$, associated with reference configuration $\kappa$, and the response function $\aleph_{\kappa_{p}}$, associated with reference configuration $\kappa_{p}$,

$$
\mathbf{T}(t)=\aleph_{\kappa}\left\{\mathbf{F}_{\kappa}(t)\right\}=\aleph_{\kappa_{p}}\left\{\mathbf{F}_{\kappa_{p}}(t)\right\}
$$

$\aleph_{\kappa_{p}}$ can be expressed in terms of $\aleph_{\kappa}$ by means of (67) and (69) as

$$
\aleph_{\kappa_{p}}\left\{\mathbf{F}_{\kappa_{p}}(t)\right\} \equiv \aleph_{\kappa}\left\{\mathbf{F}_{\kappa_{p}}(t) \overline{\mathbf{F}}_{\kappa}^{p}\right\}
$$

The form of the constitutive equation in (27) can be referred to configuration $\kappa_{p}$ by use of (67). Thus

$$
\mathbf{T}(t)=\mathbf{F}_{\kappa}(t) \overline{\boldsymbol{\beta}}_{k}\left\{\mathbf{C}_{\kappa}(t)\right\} \mathbf{F}_{\kappa}^{T}(t)=\mathbf{F}_{\kappa_{p}}(t) \overline{\boldsymbol{\beta}}_{\kappa_{p}}\left\{\mathbf{C}_{\kappa_{p}}(t)\right\} \mathbf{F}_{\kappa_{p}}^{T}(t)
$$

where $\mathbf{C}_{\kappa_{p}}(t)=\mathbf{F}_{\kappa_{p}}^{T}(t) \mathbf{F}_{\kappa_{p}}(t)$ and

$$
\overline{\boldsymbol{X}}_{\kappa_{p}}\left\{\mathbf{C}_{\kappa_{p}}(t)\right\} \equiv \overline{\mathbf{F}}_{\kappa}^{p} \overline{\boldsymbol{K}}_{\kappa}\left\{\overline{\mathbf{F}}_{\kappa}^{p T} \mathbf{C}_{\kappa_{p}}(t) \overline{\mathbf{F}}_{\kappa}^{p}\right\} \overline{\mathbf{F}}_{\kappa}^{p T}
$$

A transformation $\mathbf{H}^{\prime}$ will be a material symmetry transformation associated with $\kappa_{p}$ of the response function $\kappa_{\kappa_{p}}$ if it satisfies an equation which is analogous to (30), namely

$$
\aleph_{\kappa_{p}}\left\{\mathbf{F}_{\kappa_{p}}(t)\right\}=\aleph_{\kappa_{p}}\left\{\mathbf{F}_{\kappa_{p}}(t) \mathbf{H}^{\prime}\right\}
$$

Each transformation $\mathbf{H}^{\prime}$ for which (73) is satisfied is related to a transformation $\mathbf{H}$ for which (30) is satisfied through an application of Noll's rule [1958] (also see NEGAHBAN \& WinEMAN [1989a]). Noll's rule states that

$$
\mathbf{H}^{\prime}=\overline{\mathbf{F}}_{\kappa}^{p} \mathbf{H} \overline{\mathbf{F}}_{\kappa}^{p^{-1}}
$$

Let $g_{\alpha_{p}}^{e}(t)$ denote the group of all transformations $\mathbf{H}^{\prime}$ which satisfy (73). Therefore, Noll's rule states that $g_{\kappa_{p}}^{e}(t)=\overline{\mathbf{F}}_{\kappa}^{p} g_{\kappa}^{e}(t) \overline{\mathbf{F}}_{\kappa}^{p^{-1}}$.

In a similar manner, it can be established from (25), (27), (30), (31), and (71) that

$$
\mathbf{H}^{\prime} \overline{\boldsymbol{K}}_{\kappa_{p}}\left\{\mathbf{H}^{\prime T} \mathbf{C}_{\kappa_{p}}(t) \mathbf{H}^{\prime}\right\} \mathbf{H}^{\prime T}=\overline{\boldsymbol{K}}_{\kappa_{p}}\left\{\mathbf{C}_{\kappa_{p}}(t)\right\},
$$

where $\mathbf{H}^{\prime}=\overline{\mathbf{F}}_{\kappa}^{p} \mathbf{H} \overline{\mathbf{F}}_{\kappa}^{p^{-1}}$, for each $\mathbf{H} \in \boldsymbol{g}_{\kappa}^{e}(t)$.

These results are now applied to the two special cases treated in the previous sections. 
VIII.1. Case 1: $v_{\kappa}=\mathbf{I}$ and $\mu_{\kappa}=\mathbf{H}^{T}$

Consider a transformation $\mathbf{H} \in g_{\kappa}^{p}(t) \cap g_{\kappa}\left(t_{s}\right)$ and its corresponding transformation

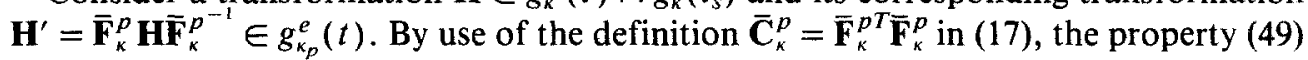
satisfied by $\mathbf{H}$ can be restated as

$$
\mathbf{H}^{T} \overline{\mathbf{F}}_{\kappa}^{p T} \overline{\mathbf{F}}_{\kappa}^{p} \mathbf{H}=\overline{\mathbf{F}}_{\kappa}^{p T} \overline{\mathbf{F}}_{\kappa}^{p}
$$

or

$$
\left(\overline{\mathbf{F}}_{\kappa}^{p-T} \mathbf{H}^{T} \overline{\mathbf{F}}_{\kappa}^{p T}\right)\left(\overline{\mathbf{F}}_{\kappa}^{p} \mathbf{H} \overline{\mathbf{F}}_{\kappa}^{p^{-1}}\right)=\mathbf{I} .
$$

This is a statement that $\mathbf{H}^{T} \mathbf{H}^{\prime}=\mathbf{I}$. In other words, each material symmetry transformation $\mathbf{H} \in g_{\kappa}^{p}(t) \cap g_{\kappa}\left(t_{s}\right)$ corresponds to a transformation $\mathbf{H}^{\prime}$ which is orthogonal. Equivalently, the material symmetry transformations of $\overline{\boldsymbol{\aleph}}_{\kappa_{p}}$, which correspond to those in $g_{\kappa}^{p}(t) \cap g_{\kappa}\left(t_{s}\right)$, form an orthogonal subgroup of $g_{\kappa_{p}}\left(t_{s}\right)=\overline{\mathbf{F}}_{\kappa}^{p} g_{\kappa}\left(t_{s}\right) \overline{\mathbf{F}}_{\kappa}^{p^{-1}}$.

VIII.2. Case 2: $\nu_{\kappa}=\mathbf{I}$ and $\mu_{\kappa}=\mathbf{I}$

Since by (53), $g_{\kappa}^{e}(t) \supseteq g_{\kappa}\left(t_{s}\right)$, it follows that $g_{\kappa_{p}}^{e}(t) \subseteq \overline{\mathbf{F}}_{\kappa}^{p} g_{\kappa}\left(t_{s}\right) \overline{\mathbf{F}}_{\kappa}^{p^{-1}}$.

In concluding this section, note that the last form in (71) is very similar to the constitutive equation proposed in some theories (like LEE [1969]). This can be seen if we write $(71)$ in the form

$$
\mathbf{T}(t)=\mathbf{F}^{e}(t) \overline{\boldsymbol{\aleph}}_{\kappa_{p}}\left\{\mathbf{C}^{e}(t)\right\} \mathbf{F}^{e T}(t)
$$

where $\mathbf{F}^{e}(t) \equiv \mathbf{F}_{\kappa_{p}}(t)$ is the "elastic deformation gradient," and $\mathbf{C}^{e}(t)=\mathbf{F}^{e T}(t) \mathbf{F}^{e}(t)$. In contrast to these theories, where the symmetries of $\mathcal{G}$ are independently prescribed, it is shown here that the symmetry properties of $\overline{\boldsymbol{X}}_{\kappa_{p}}$ cannot be independently prescribed since they are constrained by the initial symmetries of the material given by $g_{\kappa}\left(t_{s}\right)$, and other specific constitutive assumptions and the deformation history. In particular, this can present a problem when modeling initially anisotropic materials.

\section{CHANGE OF REFERENCE CONFIGURATION-ISOTROPIC MATERIALS}

The results in Section VIII are illustrated here for the case of initially isotropic materials, for which $g_{\kappa}\left(t_{s}\right)=\mathcal{O}$. Let the constitutive equation in (71) be written with respect to configuration $\kappa_{p}$ in the form given in (78), where $\mathbf{F}^{e}(t)=\mathbf{F}_{\alpha_{p}}(t)=\mathbf{F}_{\kappa}(t) \overline{\mathbf{F}}_{\kappa}^{p-1}$, and $\mathbf{C}^{e}(t)=\mathbf{F}^{e T}(t) \mathbf{F}^{e}(t)=\mathbf{C}_{\kappa_{p}}(t)$.

For this particular transformation of configuration it can be shown (see NEGAHBAN [1988]) that if we assume $\overline{\mathbf{R}}_{\kappa}^{p}=I$ for all deformation histories, then $\overline{\mathbf{R}}_{\kappa_{p}}^{p}=I$ for all deformation histories. 


\section{IX.1. Case 1: $\nu_{\kappa}=\mathbf{I}$ and $\mu_{\kappa}=\mathbf{H}^{T}$}

By use of (60) and (61), $\bar{x}_{\kappa_{p}}$ in (78) can be written as

$$
\begin{aligned}
\overline{\mathbf{B}}_{\kappa_{p}}\left\{\mathbf{C}^{e}(t)\right\}= & a_{1} \overline{\mathbf{B}}_{\kappa}^{p}+a_{2} \overline{\mathbf{B}}_{\kappa}^{p} \mathbf{C}^{e}(t) \overline{\mathbf{B}}_{\kappa}^{p}+a_{3} \overline{\mathbf{B}}_{\kappa}^{p} \mathbf{C}^{e}(t) \overline{\mathbf{B}}_{\kappa}^{p} \mathbf{C}^{e}(t) \overline{\mathbf{B}}_{\kappa}^{p} \\
& +a_{4} \overline{\mathbf{B}}_{\kappa}^{p 2}+a_{5} \overline{\mathbf{B}}_{\kappa}^{p 3}+a_{6}\left[\overline{\mathbf{B}}_{\kappa}^{p} \mathbf{C}^{e}(t) \overline{\mathbf{B}}_{\kappa}^{p 2}+\overline{\mathbf{B}}_{\kappa}^{p 2} \mathbf{C}^{e}(t) \overline{\mathbf{B}}_{\kappa}^{p}\right] \\
& +a_{7}\left[\overline{\mathbf{B}}_{\kappa}^{p} \mathbf{C}^{e}(t) \overline{\mathbf{B}}_{\kappa}^{p} \mathbf{C}^{e}(t) \overline{\mathbf{B}}_{\kappa}^{p 2}+\overline{\mathbf{B}}_{\kappa}^{p 2} \mathbf{C}^{e}(t) \overline{\mathbf{B}}_{\kappa}^{p} \mathbf{C}^{e}(t) \overline{\mathbf{B}}_{\kappa}^{p}\right] \\
& +a_{8}\left[\overline{\mathbf{B}}_{\kappa}^{p 3} \mathbf{C}^{e}(t) \overline{\mathbf{B}}_{\kappa}^{p}+\overline{\mathbf{B}}_{\kappa}^{p} \mathbf{C}^{e}(t) \overline{\mathbf{B}}_{\kappa}^{p 3}\right] \\
& +a_{9}\left[\overline{\mathbf{B}}_{\kappa}^{p} \mathbf{C}^{e}(t) \overline{\mathbf{B}}_{\kappa}^{p} \mathbf{C}^{e}(t) \overline{\mathbf{B}}_{\kappa}^{p 3}+\overline{\mathbf{B}}_{\kappa}^{p 3} \mathbf{C}^{e}(t) \overline{\mathbf{B}}_{\kappa}^{p} \mathbf{C}^{e}(t) \overline{\mathbf{B}}_{\kappa}^{p}\right]
\end{aligned}
$$

In this equation, $a_{1}, \ldots, a_{9}$ are functions of the three isotropic invariants of $\mathbf{C}_{k}(t)=$ $\overline{\mathbf{F}}_{k}^{p T} \mathbf{C}^{e}(t) \overline{\mathbf{F}}_{k}^{p}$, and the four mixed isotropic invariants of $\mathbf{C}_{k}(t)$ and $\overline{\mathbf{C}}_{\kappa}^{p}$ (given in (59)), and $\overline{\mathbf{B}}_{\kappa}^{p}=\overline{\mathbf{F}}_{\kappa}^{p} \overline{\mathbf{F}}_{\kappa}^{p T}$. The functions $a_{1}, \ldots, a_{9}$ can be also written in terms of the invariants expressed in terms of $\mathbf{C}^{e}(t)$. In this form, $\overline{\boldsymbol{K}}_{\kappa_{p}}$ will have the property

$$
\mathbf{H}^{\prime} \overline{\boldsymbol{\aleph}}_{\kappa_{p}}\left\{\mathbf{H}^{\prime T} \mathbf{C}^{e}(t) \mathbf{H}^{\prime}\right\} \mathbf{H}^{\prime T}=\overline{\boldsymbol{\aleph}}_{\kappa_{p}}\left\{\mathbf{C}^{e}(t)\right\}
$$

which holds, at least, for $\mathbf{H}^{\prime} \in \overline{\mathbf{F}}_{\kappa}^{p}\left[g_{\kappa}^{p} \cap g_{\kappa}\left(t_{s}\right)\right] \overline{\mathbf{F}}_{\kappa}^{p^{-1}}$.

It was shown in Section VII that the transformations representing the symmetries of the current elastic response for an initially isotropic material represent one of three possible symmetries (i.e., isotropy, transverse isotropy, or orthotropy). As shown above, the symmetry groups $\overline{\mathbf{F}}_{\kappa}^{p}\left[g_{\kappa}^{p} \cap g_{\kappa}\left(t_{s}\right)\right] \overline{\mathbf{F}}_{\kappa}^{p^{-1}}$ are simply conjugate (through Noll's rule [1958]) to the groups given in Section VII.

\section{IX.2. Case 2: $\nu_{\kappa}=$ I and $\mu_{\kappa}=\mathbf{I}$}

If (65) is written with respect to configuration $\kappa_{p}$, the constitutive equation for the current elastic response will have the form in (78) where

$$
\overline{\boldsymbol{\aleph}}_{\kappa}\left\{\mathbf{C}^{e}(t)\right\}=r_{1} \overline{\mathbf{B}}_{\kappa}^{p}+r_{2} \overline{\mathbf{B}}_{\kappa}^{p} \mathbf{C}^{e}(t) \overline{\mathbf{B}}_{\kappa}^{p}+r_{3} \overline{\mathbf{B}}_{\kappa}^{p} \mathbf{C}^{e}(t) \overline{\mathbf{B}}_{\kappa}^{p} \mathbf{C}^{e}(t) \overline{\mathbf{B}}_{\kappa}^{p}
$$

The function $\overline{\boldsymbol{K}}_{\kappa_{p}}$ will satisfy (80) for every $\mathbf{H}^{\prime} \in \overline{\mathbf{F}}_{\kappa}^{p} \mathcal{O} \overline{\mathbf{F}}_{\kappa}^{p^{-1}}$. As has been discussed in Negahban and Wineman [1989a] and WiNEMAN et al. [1988], the group $\overline{\mathbf{F}}_{\kappa}^{p} \mathcal{O} \overline{\mathbf{F}}_{\kappa}^{p^{-1}}$ contains both orthogonal and unimodular non-orthogonal elements. This is in strong contrast to the results for case 1 , in which $\overline{\mathbf{F}}_{\kappa}^{p}\left[g_{\kappa}^{p} \cap g_{k}\left(t_{s}\right)\right] \overline{\mathbf{F}}_{\kappa}^{p^{-1}}$ contained only orthogonal elements. It is worth noting that the orthogonal elements of $\overline{\mathbf{F}}_{k}^{p} \mathcal{O} \overline{\mathbf{F}}_{k}^{p^{-1}}$ coincide with those of $\overline{\mathbf{F}}_{\kappa}^{p}\left[g_{\kappa}^{p} \cap g_{\kappa}\left(t_{s}\right)\right] \overline{\mathbf{F}}_{\kappa}^{p^{-1}}$ of case 1, and, therefore, describe isotropy, transverse isotropy, and orthotropy, depending on the principal values and vectors of $\overline{\mathbf{B}}_{\kappa}^{p}$ in the same manner as in case 1. It is also worth mentioning that the unimodular non-orthogonal members of $\overline{\mathbf{F}}_{\kappa}^{p} \mathcal{O \overline { \mathbf { F } } _ { \kappa } ^ { p }}{ }^{-1}$ impose further restrictions on the form of the constitutive equation for the present case. These additional symmetries are responsible for the differences between (81) and (79). 


\section{VISCOELASTIC-PLASTIC MATERIALS}

Consider a viscoelastic-plastic material as discussed in NEGAHBAN [1988]. The constitutive equation is given by

$$
\mathbf{T}(t)=\mathcal{T}\left\{\begin{array}{c}
t \\
\mathbf{F}(s) \\
s=t_{s}
\end{array} ; \mathbf{F}^{p}(t)\right\}
$$

where $\mathrm{F}^{p}(t)$ is given by an equation similar to (4) and the notation implies that the range of deformation gradients needed to evaluate the viscoelastic response is given as all $\mathbf{F}(s)$ for $s \in\left(t_{s}, t\right]$. The constraint of frame-indifference is satisfied by the introduction of the alternate form of (82) as

$$
\mathbf{T}(t)=\mathbf{F}(t) \mathcal{S}\left\{\begin{array}{c}
t \\
\mathbf{C}(s) \\
s=t_{s}
\end{array} ; \overline{\mathbf{F}}^{p}(t)\right\} \mathbf{F}^{T}(t)
$$

where $\overline{\mathbf{F}}^{p}(t)$ is given in (6). An alternate form for this can be written as

$$
\mathbf{T}(t)=\mathbf{F}(t) \overline{\mathrm{s}}\left\{\begin{array}{c}
t \\
\mathbf{C}(s) \\
s=t_{s}
\end{array} ; \overline{\mathbf{R}}^{p}(t) ; \overline{\mathbf{C}}^{p}(t)\right\} \mathbf{F}^{T}(t),
$$

where $\overline{\mathbf{R}}^{p}(t)$ and $\overline{\mathbf{C}}^{p}(t)$ are the orthogonal and the square of the right symmetric part of $\overline{\mathbf{F}}^{p}(t)$ in its polar decomposition, respectively, and given in (14) and (17).

The existence of symmetry in the response of the material at the starting time $t_{s}$ requires that the functional $S$ satisfy the condition

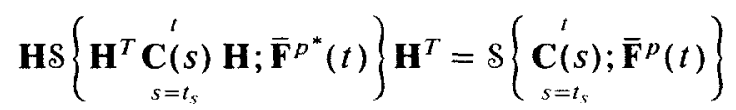

for every $\mathbf{H} \in g\left(t_{s}\right)$, and where $\overrightarrow{\mathbf{F}}^{p^{*}}(t)$ is given in (12). This restriction applied to the alternate form given in $(84)$ will require that

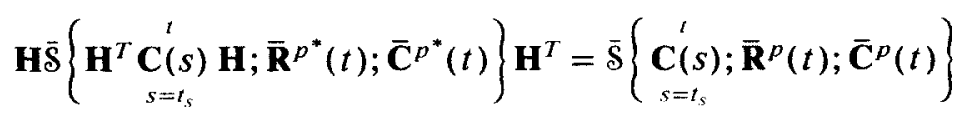

for every $\mathbf{H} \in g\left(t_{s}\right)$, and where $\overline{\mathbf{R}}^{p^{*}}(t)$ and $\overline{\mathbf{C}}^{p^{*}}(t)$ are given in (19) and (20).

Henceforth we will assume that relative to configuration $\kappa$ the value of $\overline{\mathbf{R}}^{p}(t)=\mathbf{I}$ for all deformation histories. Let

$$
\mathbf{T}(t)=\mathbf{F}(t) \overline{\bar{x}}\left\{\begin{array}{c}
t \\
\mathbf{C}(s) \\
s=t_{s}
\end{array}\right\} \mathbf{F}^{T}(t)
$$

give the current viscoelastic response functional of the material within a given yield surface. The functional $\bar{k}$ is given in terms of $\overline{\mathcal{S}}$ as

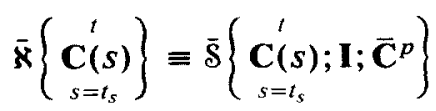


for which the fixed value of $\overline{\mathbf{C}}^{p}(t)$ within the yield surface under consideration is denoted by $\overline{\mathbf{C}}^{p}$.

The symmetries of the current viscoelastic response can be evaluated through finding every $\mathbf{H}$ which satisfies the condition

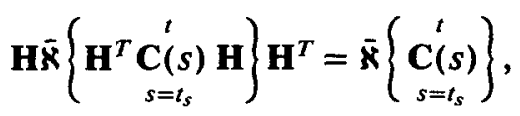

irrespective of the actual history of $\mathbf{C}(s)$ for $s \in\left(t_{s}, t\right]$. Equation (89) can be written in terms of the functional $\bar{S}$ through (88), and is

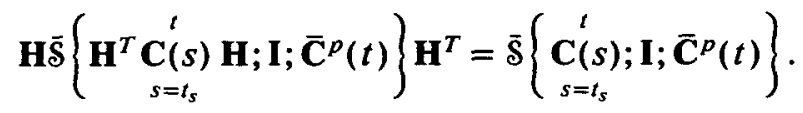

Comparing (90) to the restrictions imposed on the material by its symmetries at the starting time and given in (86), the solution to the problem of finding the symmetries of the current viscoelastic response can be stated as

$$
g^{v e}(t) \supseteq g\left(t_{s}\right) \cap g^{p}(t)
$$

where $g^{v e}(t)$ is the group of transformations which represent the symmetries of the current viscoelastic response and $g^{p}(t)$ is given in (37).

It is seen that the evolution of the symmetries in the current viscoelastic response of a viscoelastic-plastic material is given by an equation which is identical to that for the evolution of the current elastic response in an elastic-plastic material. Thus, all statements made about the symmetries of the current elastic response of the elastic-plastic material, given in Section V, can be directly restated for the current viscoelastic response of the viscoelastic-plastic material.

\section{REFERENCES}

1955 Rivlin, R.S., and ERICKSON, J.L., "Stress-Deformation Relations for Isotropic Materials," J. Rational Mech. Anal., 4 (2), 325.

1958 NoLl, W., “A Mathematical Theory of the Mechanical Behavior of Continuous Media," Arch. Rational Mech. Anal., 2, 197.

1964 Wineman, A.S., and PIPKIN, A.C., "Material Symmetry Restrictions on Constitutive Equations," Arch. Rational Mech. Anal., 17, 184.

1969 LEE, E.H., "Elastic-Plastic Deformation at Finite Strain," J. Appl. Mech., 36, 1.

1975 Spencer, A.J.M., "Theory of Invariants," in Eringen, A.C. (ed.), Continuum Physics, Vol. 1, Academic Press, New York.

1980 CASEY, J., and NAGHDI, P.M., "A Remark on the Use of the Decomposition $F=F_{e} F_{p}$ in Plasticity," Journal of Applied Mechanics, 47, 672.

1981 CaSEY, J., and NAGHDI, P.M., "A Correct Definition of Elastic and Plastic Deformation and Its Computational Significance," Journal of Applied Mechanics, 48, 983.

1988 Negahban, M., "Material Symmetry and the Evolution of Anisotropies in First Gradient Theories of Nonlinear Material Behavior; Inelasticity; Polymer Crystallization," Ph.D. Dissertation, University of Michigan.

1988 Wineman, A.S., Rajagopal, K.R., and Negahban, M. "Changes in Material Symmetry Associated with Deformation: Uniaxial Extension," Int. J. Eng. Sci., 26 (12), 1307.

1989a Negahban, M., and Wineman, A.S., "Material Symmetry and the Evolution of Anisotropies in a Simple Material: I. Change of Reference Configuration," Int. J. Nonlinear Mech., 24 (6) 521. 
1989b Negahban, M., and Wineman, A.S., "Material Symmetry and the Evolution of Anisotropies in a Simple Material: II. The Evolution of Symmetry," Int. J. Nonlinear Mech., 24 (6), 537.

Department of Engineering Mechanics and The Center for Materials Research and Analysis University of Nebraska-Lincoln

Lincoln, NE 68588-0347, USA

Department of Mechanical Engineering and Applied Mechanics

The University of Michigan

Ann Arbor, MI 48109, USA

(Received 8 August 1989; in final revised form 15 June 1991)

\section{APPENDIX}

\section{YIELD FUNCTION AND HARDENING PARAMETER}

The yield function in (21) must be subjected to the constraints of frame indifference and material symmetry. Consider first the restriction of frame indifference. For two histories which differ by rigid body motions, the corresponding deformation gradients are $\mathbf{F}(s)$ and $\mathbf{Q}(s) \mathbf{F}(s)$, where $\mathbf{Q}(s) \in \mathcal{O}$ for each $s(\mathcal{\theta}$ is the group of linear orthogonal transformations). We assume that the restriction of frame indifference on the yield function requires that:

$$
f\left\{\mathbf{F}(t) ; \mathbf{F}^{p}(t) ; k(t)\right\}=f\left\{\mathbf{Q}(t) \mathbf{F}(t) ; \hat{\mathbf{F}}^{p}(t) ; \hat{k}(t)\right\}
$$

where

$$
\hat{\mathbf{F}}^{p}(t)=\mathcal{F}^{p}\{\mathbf{Q}(s) \underset{\mathbf{F}(s)}{t}\},
$$

and

$$
\hat{k}(t)=k\left\{\mathbf{Q}(s) \begin{array}{c}
\stackrel{t}{\mathbf{F}}(s) \\
s=t_{s}
\end{array}\right\} .
$$

Letting $\mathbf{Q}(s)=\mathbf{R}^{T}(s)$, the orthogonal part of the polar decomposition of $\mathbf{F}(s)$, restriction $(92)$, requires

$$
\begin{aligned}
& f\left\{\mathbf{F}(t) ; \mathbf{F}^{p}(t)=\mathcal{F}^{p}\left\{\begin{array}{c}
t \\
\mathbf{F}(s) \\
s=t_{s}
\end{array}\right\} ; k(t)\right\} \\
& =f\left\{\mathbf{U}(t) ; \hat{\mathbf{F}}^{p}(t)=\mathcal{F}^{p}\left\{\begin{array}{c}
t \\
\mathbf{U}(s) \\
s=t_{s}
\end{array}\right\} ; \hat{k}(t)=k\left\{\begin{array}{c}
t \\
\mathbf{U}(s) \\
s=t_{s}
\end{array}\right\}\right\} .
\end{aligned}
$$

This can be written as

$$
f\left\{\mathbf{F}(t) ; \mathbf{F}^{p}(t) ; k(t)\right\}=\vec{f}\left\{\mathbf{C}(t) ; \overline{\mathbf{F}}^{p}(t) ; \bar{k}(t)\right\},
$$


where $\overline{\mathbf{F}}^{p}(t)$ is given by (6), $\mathbf{C}(t)$ is defined in (7), and $\bar{k}(t)$ represents the value of $k$ evaluated for the history of deformation gradients $\mathbf{U}(s)$ derived from the actual history $\mathbf{F}(s)$. As in Section III, we can represent $\overline{\mathbf{F}}^{p}(t)$ by the products of its polar decomposition and introduce the alternate form

$$
f\left\{\mathbf{F}(t) ; \mathbf{F}^{p}(t) ; k(t)\right\}=\bar{f}\left\{\mathbf{C}(t) ; \overline{\mathbf{R}}^{p}(t) ; \overline{\mathbf{C}}^{p}(t) ; \bar{k}(t)\right\}
$$

where $\overline{\mathbf{R}}^{p}(t)$ and $\overline{\mathbf{C}}^{p}(t)$ are defined in (14), (17), respectively.

We assume the restriction of material symmetry to require that

$$
f\left\{\mathbf{F}(t) ; \mathbf{F}^{p}(t) ; k(t)\right\}=f\left\{\mathbf{F}(t) \mathbf{H} ; \mathbf{F}^{p^{*}}(t) ; k^{*}(t)\right\},
$$

for every $\mathbf{H} \in g\left(t_{s}\right)$, and where the "* " indicates that the functional for the parameter is evaluated for the history $\mathbf{F}(s) \mathbf{H}$. By using (97), this can be restated as

$$
\bar{f}\left\{\mathbf{C}(t) ; \overline{\mathbf{R}}^{p}(t) ; \overline{\mathbf{C}}^{p}(t) ; \bar{k}(t)\right\}=\bar{f}\left\{\mathbf{H}^{T} \mathbf{C}(t) \mathbf{H} ; \overline{\mathbf{R}}^{p^{*}}(t) ; \overline{\mathbf{C}}^{p^{*}}(t) ; \bar{k}^{*}(t)\right\},
$$

where $\overline{\mathbf{R}}^{p^{*}}(t)$ and $\overline{\mathbf{C}}^{p^{*}}(t)$ are defined by (19) and (20), respectively. We will henceforth assume that $\bar{k}(t)$ follows the material symmetry restriction

$$
\left.\bar{k}(t)=\bar{k}_{\kappa}\left\{\begin{array}{c}
\mathbf{C}^{(}(s) \\
s=t_{s}
\end{array}\right\}=\bar{k}_{\kappa}\left\{\mathbf{H}^{T} \underset{\mathbf{C}(s) \mathbf{H}}{s=t_{s}}\right\}\right\} .
$$

\section{A.1. Initially isotropic materials}

For materials that are initially isotropic, $g\left(t_{s}\right)=\mathcal{O}$.

A.1.1. Case 1: $\nu=\mathbf{I}$ and $\mu=\mathbf{H}^{T}$. The material symmetry restriction on the yield function, stated in (99), becomes, in view of the assumptions of the present case,

$$
\bar{f}\left\{\mathbf{H}^{T} \mathbf{C}(t) \mathbf{H} ; \mathbf{I} ; \mathbf{H}^{T} \overline{\mathbf{C}}^{p}(t) \mathbf{H} ; \bar{k}(t)\right\}=\bar{f}\left\{\mathbf{C}(t) ; \mathbf{I} ; \overline{\mathbf{C}}^{p}(t) ; \bar{k}(t)\right\},
$$

for every $\mathbf{H} \in g\left(t_{s}\right)=\mathcal{O}$. Therefore, $\bar{f}$ will be an isotropic scalar function of the two symmetric tensors $\mathbf{C}(t)$ and $\overline{\mathbf{C}}^{p}(t)$. This results in $f(t)$ given by

$$
f(t)=\bar{f}\left\{I_{1}^{*}, \ldots, I_{10}^{*} ; \bar{k}(t)\right\}
$$

where $I_{1}^{*}, \ldots, I_{10}^{*}$ are given in (59).

The constraints given in (100) requires that $\bar{k}(t)$ be an isotropic scalar functional of the history of $\mathrm{C}(s)$ which can be written as

$$
\bar{k}(t)=\bar{k}_{\kappa}\left\{I_{1}, \ldots, I_{6}\right\}
$$

for $I_{1}, \ldots, I_{6}$ given in (57). 
A.1.2. Case 2: $\nu=\mathbf{I}$ and $\mu=\mathbf{I}$. In this case, the material symmetry restriction on the yield function is given as

$$
\bar{f}\left\{\mathbf{H}^{T} \mathbf{C}(t) \mathbf{H} ; \mathbf{I} ; \overline{\mathbf{C}}^{p}(t) ; \bar{k}(t)\right\}=\bar{f}\left\{\mathbf{C}(t) ; \mathbf{I} ; \overline{\mathbf{C}}^{p}(t) ; \bar{k}(t)\right\},
$$

for every $\mathbf{H} \in g\left(t_{s}\right)=\mathcal{O}$. Therefore, we can write

$$
f(t)=\bar{f}\left\{I_{1}^{*}, I_{2}^{*}, I_{3}^{*} ; \overline{\mathbf{C}}^{p}(t) ; \bar{k}(t)\right\},
$$

where $I_{1}^{*}, I_{2}^{*}$, and $I_{3}^{*}$ are the three isotropic invariants of $\mathbf{C}(t)$.

The constraint of material symmetry on $k(t)$ is identical to the first case and is given in (100) and these constraints result in the same equation as is given in (103). 